\title{
A Concise Total Synthesis of (+)-FR900482 and (+)-FR66979
}

\author{
Ted C. Judd and Robert M. Williams* \\ e-mail: rmw@chem.colostate.edu \\ Contribution from the Department of Chemistry \\ Colorado State University, Fort Collins, Colorado 80523
}

\section{Supporting Information}

\section{Table of contents}

(1) N-para-methoxybenzylation of $\mathbf{1 0}$

Page number

(2) Preparation of $\mathbf{1 5}$

(3) Oxidation of $\mathbf{1 5}$

(4) Aldol condensation of 15: preparation of $\mathbf{1 1}$ and $\mathbf{1 6}$

(5) Epimerization of $\mathbf{1 6}$ to $\mathbf{1 1}$

(6) Preparation of 20

(7) Preparation of 22

S4

(8) Silyl ether cleavage of $\mathbf{2 2}$

S5

(9) Preparation of $\mathbf{2 5}$

S6

(10) Preparation of 26

(11) Preparation of 2

(12) Preparation of 1

S7

S8

(1aS,9aS)-Dimethyl ester-1a,2,3,8,9,9a-hexahydro-7-(methoxymethoxy)-3-[(4methoxyphenyl)methyl]-9-[[diethyl(1-methylethyl)silyl]oxy]-1H-azirino[2,3-c][1]benzazocine1,5-dicarboxylic acid.
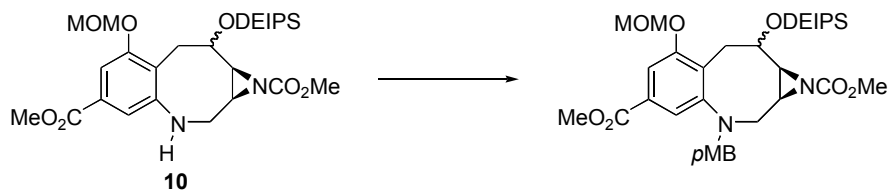

Aniline 10 was prepared as described previously ${ }^{1}(200 \mathrm{mg}, 0.404 \mathrm{mmol}, 1.0$ equiv.) was dissolved in 6 $\mathrm{mL}$ of dry $\mathrm{CH}_{2} \mathrm{Cl}_{2}$. DIPEA (211 $\mu \mathrm{L}, 1.21 \mathrm{mmol}, 3.0$ equiv.) was added followed by dropwise addition of PMB-Br (106 $\mu \mathrm{L}, 0.525 \mathrm{mmol}, 1.3$ eq.). The solution was allowed to stir $48 \mathrm{~h}$ until TLC analyses (2:1 $\mathrm{Hex} / \mathrm{EtOAc}$ ) showed loss of starting material. The reaction was quenched by the addition of sat $\mathrm{NaHCO}_{3}$ (aq). The layers were separated, and the aqueous layer was extracted $3 \mathrm{X} \mathrm{CH}_{2} \mathrm{Cl}_{2}$. The combined organic layers were concentrated, and the crude material was taken up in a 2:1 Hex/EtOAc solution, washed $2 \mathrm{X}$ $\mathrm{H}_{2} \mathrm{O}, 1 \mathrm{X} \mathrm{NaCl}_{(\mathrm{aq})}$ and dried over $\mathrm{Na}_{2} \mathrm{SO}_{4}$. Following concentration, the crude oil was purified by column chromatography $(3: 1 \mathrm{Hex} / \mathrm{EtOAc})$ to afford $215 \mathrm{mg}(86 \%$ yield $)$ of the PMB protected aniline as a colorless oil. 
Major Diastereomerer: $[\alpha]_{\mathrm{D}}^{25}=+77.1\left(\mathrm{c}=1.0, \mathrm{CHCl}_{3}\right){ }^{1} \mathrm{H}$ NMR $\left(300 \mathrm{MHz}, \mathrm{CDCl}_{3}\right) \delta \mathrm{CHCl}_{3}: 0.70-0.77$ $(\mathrm{m}, 4 \mathrm{H}), 1.00-1.04(\mathrm{~m}, 13 \mathrm{H}), 2.23(\mathrm{dd}, \mathrm{J}=6.0 \mathrm{~Hz}, 12.0 \mathrm{~Hz}, 1 \mathrm{H}), 2.49(\mathrm{dd}, \mathrm{J}=4.5 \mathrm{~Hz}, 6.0 \mathrm{~Hz}, 1 \mathrm{H})$, 3.07 (dd, J = 10.5 Hz, 13.5 Hz, 1 H), 3.18 (dd, J = 4.5 Hz, 13.5 Hz, 1 H), 3.46 (m, 2 H), 3.50 (s, $3 \mathrm{H}), 3.65$ $(\mathrm{s}, 3 \mathrm{H}), 3.80(\mathrm{~s}, 3 \mathrm{H}), 3.89(\mathrm{~s}, 3 \mathrm{H}), 4.33(1 / 2 \mathrm{ABq}, \mathrm{J}=13.5 \mathrm{~Hz}, 1 \mathrm{H}), 4.41(1 / 2 \mathrm{ABq}, \mathrm{J}=13.5 \mathrm{~Hz}, 1 \mathrm{H})$, $4.55(\mathrm{~m}, 1 \mathrm{H}), 5.21(1 / 2 \mathrm{ABq}, \mathrm{J}=7.5 \mathrm{~Hz}, 1 \mathrm{H}), 5.26(1 / 2 \mathrm{ABq}, \mathrm{J}=7.5 \mathrm{~Hz}, 1 \mathrm{H}), 6.85(\mathrm{~d}, \mathrm{~J}=9.0 \mathrm{~Hz}, 2 \mathrm{H})$, $7.27(\mathrm{~d}, \mathrm{~J}=9.0 \mathrm{~Hz}, 2 \mathrm{H}), 7.38(\mathrm{~d}, \mathrm{~J}=1.5 \mathrm{~Hz}, 1 \mathrm{H}), 7.47(\mathrm{~d}, \mathrm{~J}=1.5 \mathrm{~Hz}, 1 \mathrm{H}) ;{ }^{13} \mathrm{C}$ NMR $\left(75 \mathrm{MHz}, \mathrm{CDCl}_{3}\right)$

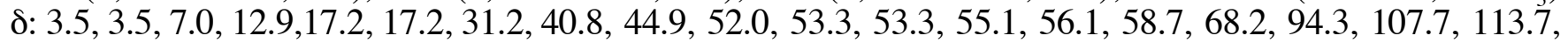
113.7, 115.0, 125.3, 129.0, 129.6, 129.6, 130.3, 151.7, 155.4, 158.7, 163.6, 166.8; IR (NaCl/neat) 2952, $1733,1718,1653,1576,1559 \mathrm{~cm}^{-1}$; HRMS (FAB+) calcd for $\mathrm{C}_{32} \mathrm{H}_{47} \mathrm{~N}_{2} \mathrm{O}_{8} \mathrm{Si}_{1}(\mathrm{~m} / \mathrm{z}) 615.3102$, found $(\mathrm{m} / \mathrm{z})$ 615.3094.

Minor Diastereomer: $[\alpha]_{\mathrm{D}}^{25}=+83.9\left(\mathrm{c}=0.87, \mathrm{CHCl}_{3}\right){ }^{1} \mathrm{H}$ NMR $\left(300 \mathrm{MHz}, \mathrm{CDCl}_{3}\right) \delta \mathrm{CHCl}_{3}: 0.69-0.74$ $(\mathrm{m}, 4 \mathrm{H}), 0.99-1.07(\mathrm{~m}, 13 \mathrm{H}), 2.22(\mathrm{dd}, \mathrm{J}=6.0 \mathrm{~Hz}, 13.5 \mathrm{~Hz}, 1 \mathrm{H}), 2.51(\mathrm{dd}, \mathrm{J}=7.5 \mathrm{~Hz}, 7.5 \mathrm{~Hz}, 1 \mathrm{H})$, $2.96(\mathrm{dd}, \mathrm{J}=6.0 \mathrm{~Hz}, 12.0 \mathrm{~Hz}, 1 \mathrm{H}), 3.04(\mathrm{dd}, \mathrm{J}=6.0 \mathrm{~Hz}, 15.0 \mathrm{~Hz}, 1 \mathrm{H}), 3.27(\mathrm{~m}, 2 \mathrm{H}), 3.50(\mathrm{~s}, 3 \mathrm{H}), 3.62(\mathrm{~s}$, $3 \mathrm{H}), 3.79(\mathrm{~s}, 3 \mathrm{H}), 3.91(\mathrm{~s}, 3 \mathrm{H}), 4.06(1 / 2 \mathrm{ABq}, \mathrm{J}=12 \mathrm{~Hz}, 2 \mathrm{H}), 4.14(1 / 2 \mathrm{ABq}, \mathrm{J}=12 \mathrm{~Hz}, 2 \mathrm{H}), 4.41(\mathrm{~m}, 1$ $\mathrm{H}), 5.20(1 / 2 \mathrm{ABq}, \mathrm{J}=7.5 \mathrm{~Hz}, 1 \mathrm{H}), 5.25(1 / 2 \mathrm{ABq}, \mathrm{J}=7.5 \mathrm{~Hz}, 1 \mathrm{H}), 6.80(\mathrm{~d}, \mathrm{~J}=9.0 \mathrm{~Hz}, 2 \mathrm{H}), 7.23(\mathrm{~d}, \mathrm{~J}=$

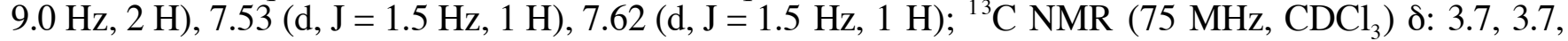
7.0, 7.1,13.0, 17.3, 17.3, 34.3, 38.6, 47.7, 52.2, 53.3, 55.2, 56.3, 56.6, 59.5, 71.6, 94.7, 111.4, 113.6, 113.6, 117.5, 129.2, 129.9, 129.9, 130.3, 131.8, 152.1, 155.4, 158.6, 163.2, 166.6; IR (NaCl/neat) 2952, 1726, $1512 \mathrm{~cm}^{-1}$; HRMS (FAB+) calcd for $\mathrm{C}_{32} \mathrm{H}_{47} \mathrm{~N}_{2} \mathrm{O}_{8} \mathrm{Si}_{1}(\mathrm{~m} / z) 615.3102$, found $(\mathrm{m} / z) 615.3080$.

(1aS,9aS)-Dimethyl ester -1a,2,3,8,9,9a-hexahydro-7-(methoxymethoxy)-3-[(4methoxyphenyl)methyl]-9-hydroxy -1H-azirino[2,3-c][1]benzazocine-1,5-dicarboxylic acid. (15)
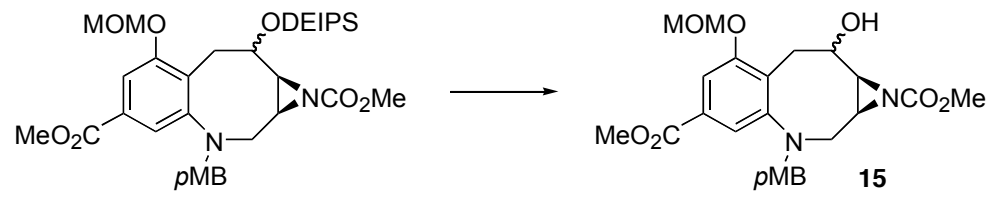

To a solution of the silyl ether from the previous reaction (760 mg, $1.23 \mathrm{mmol}, 1.0$ equiv.) in $16 \mathrm{~mL}$ of a 10:1 solution of $\mathrm{DMF} / \mathrm{H}_{2} \mathrm{O}$ was added Tris(dimethylamino)sulfur (trimethylsilyl)difluoride (TASF) (700 $\mathrm{mg}, 2.54 \mathrm{mmol}, 2.1$ equiv.). After stirring at ambient temperature for $4 \mathrm{~h}$, an additional portion of TASF (350 mg, $1.27 \mathrm{mmol}, 1.0$ equiv.) was added and the reaction was continued for $2 \mathrm{~h}$ until TLC analyses (2:1 $\mathrm{CH}_{2} \mathrm{Cl}_{2} / \mathrm{Et}_{2} \mathrm{O}$ ) showed complete consumption of the starting material. Addition of saturated $\mathrm{NH}_{4} \mathrm{Cl}_{(\mathrm{aq})}$ followed by dilution with EtOAC and $\mathrm{H}_{2} \mathrm{O}$ afforded a biphasic mixture that was subsequently separated. Extraction of the aqueous phase with $3 \mathrm{X}$ EtOAc followed by washing the combined organic extracts $1 \mathrm{X}$ brine and drying over $\mathrm{Na}_{2} \mathrm{SO}_{4}$ afforded a crude oil that was dried under vacuum overnight. Purification of the crude material by column chromatography $\left(2: 1 \mathrm{CH}_{2} \mathrm{Cl}_{2} / \mathrm{Et}_{2} \mathrm{O}\right)$ furnished $480 \mathrm{mg}$ of the alcohol 15 (80\% yield) as a white crystalline solid following concentration in $\mathrm{Et}_{2} \mathrm{O}$.

Major Diastereomer: $[\alpha]_{\mathrm{D}}^{25}=+105.1\left(\mathrm{c}=1.0, \mathrm{CHCl}_{3}\right)$ m.p. $48-50{ }^{\circ} \mathrm{C}{ }^{1} \mathrm{H}$ NMR $\left(300 \mathrm{MHz}, \mathrm{CDCl}_{3}\right) \delta$ $\mathrm{CHCl}_{3}: 2.40(\mathrm{~m}, 1 \mathrm{H}), 2.52(\mathrm{dd}, \mathrm{J}=4.5 \mathrm{~Hz}, 6.0 \mathrm{~Hz}, 1 \mathrm{H}), 2.79(\mathrm{dd}, \mathrm{J}=7.5 \mathrm{~Hz}, 15.0 \mathrm{~Hz}, 1 \mathrm{H}), 3.30$ (dd, J = $7.5 \mathrm{~Hz}, 13.5 \mathrm{~Hz}, 1 \mathrm{H}), 3.49(\mathrm{~s}, 3 \mathrm{H}), 3.51(\mathrm{~m}, 2 \mathrm{H}), 3.65(\mathrm{~s}, 3 \mathrm{H}), 3.78(\mathrm{~s}, 3 \mathrm{H}), 3.89$ (s, $3 \mathrm{H}), 4.26(1 / 2$ $\mathrm{ABq}, \mathrm{J}=13.5 \mathrm{~Hz}, 2 \mathrm{H}), 4.39(1 / 2 \mathrm{ABq}, \mathrm{J}=13.5 \mathrm{~Hz}, 2 \mathrm{H}), 4.55(\mathrm{~m}, 1 \mathrm{H}), 5.24($ ap. s, $2 \mathrm{H}), 6.82(\mathrm{~d}, \mathrm{~J}=9.0$ $\mathrm{Hz}, 2 \mathrm{H}), 7.21(\mathrm{~d}, \mathrm{~J}=9.0 \mathrm{~Hz}, 2 \mathrm{H}), 7.41(\mathrm{~d}, \mathrm{~J}=1.5 \mathrm{~Hz}, 1 \mathrm{H}), 7.58(\mathrm{~d}, \mathrm{~J}=1.5 \mathrm{~Hz}, 1 \mathrm{H}) ;{ }^{13} \mathrm{C}$ NMR $(75$

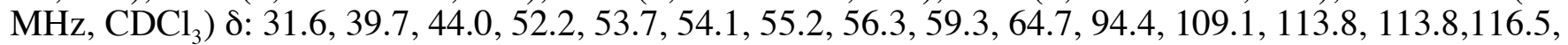
126.8, 129.4, 129.6, 129.6, 150.8, 155.2, 158.8, 163.2, 166.5; IR (NaCl/neat) 3462, 2953, 1720, 1576, 1512 $\mathrm{cm}^{-1}$; HRMS (FAB+) calcd for $\mathrm{C}_{25} \mathrm{H}_{31} \mathrm{~N}_{2} \mathrm{O}_{8}(\mathrm{~m} / z)$ 487.2080, found $(\mathrm{m} / z)$ 487.2068.

Minor Diastereomer: $[\alpha]_{\mathrm{D}}^{25}=+103.6\left(\mathrm{c}=0.87, \mathrm{CHCl}_{3}\right)$ m.p. $57-59{ }^{\circ} \mathrm{C}{ }^{1} \mathrm{H}$ NMR $\left(300 \mathrm{MHz}, \mathrm{CDCl}_{3}\right) \delta$ $\mathrm{CHCl}_{3}: 2.29(\mathrm{~m}, 1 \mathrm{H}), 2.95(\mathrm{dd}, \mathrm{J}=7.5 \mathrm{~Hz}, 13.5 \mathrm{~Hz} 1 \mathrm{H}), 3.08(\mathrm{dd}, \mathrm{J}=6.0 \mathrm{~Hz}, \mathrm{~J}=15.0 \mathrm{~Hz}, 1 \mathrm{H}), 3.13(\mathrm{~d}$, $\mathrm{J}=4.5 \mathrm{~Hz}, 1 \mathrm{H}), 3.23(\mathrm{dd}, \mathrm{J}=6.0 \mathrm{~Hz}, \mathrm{~J}=15.0 \mathrm{~Hz}, 1 \mathrm{H}), 3.39(\mathrm{~m}, 1 \mathrm{H}), 3.49(\mathrm{~s}, 3 \mathrm{H}), 3.62(\mathrm{~s}, 3 \mathrm{H}), 3.77(\mathrm{~s}$, $3 \mathrm{H}), 3.90(\mathrm{~s}, 3 \mathrm{H}), 4.13(1 / 2 \mathrm{ABq}, \mathrm{J}=13.5,1 \mathrm{H}), 4.23(1 / 2 \mathrm{ABq}, \mathrm{J}=13.5,1 \mathrm{H}), 4.44(\mathrm{~m}, 1 \mathrm{H}), 5.25$ (ap. s, $2 \mathrm{H}), 6.81(\mathrm{~d}, \mathrm{~J}=9.0 \mathrm{~Hz}, 2 \mathrm{H}), 7.20(\mathrm{~d}, \mathrm{~J}=9.0 \mathrm{~Hz}, 2 \mathrm{H}), 7.48(\mathrm{~d}, \mathrm{~J}=1.5 \mathrm{~Hz}, 1 \mathrm{H}), 7.61(\mathrm{~d}, \mathrm{~J}=1.5 \mathrm{~Hz}, 1$ 
$\mathrm{H}) ;{ }^{13} \mathrm{C} \mathrm{NMR}\left(75 \mathrm{MHz}, \mathrm{CDCl}_{3}\right)$ $\delta: 31.8,38.8,47.4,52.2,53.6,55.1,55.4,56.4,59.4,71.2,94.7,110.4$, 113.7, 113.7, 117.4, 129.4, 129.7, 129.7, 130.0, 151.8, 155.1, 158.6, 163.0, 166.4; IR (NaCl/neat) 2953, 1718, 1701, 1684, $1653 \mathrm{~cm}^{-1}$; HRMS (FAB+) calcd for $\mathrm{C}_{25} \mathrm{H}_{31} \mathrm{~N}_{2} \mathrm{O}_{8}(\mathrm{~m} / z) 487.2080$, found $(\mathrm{m} / z)$ 487.2083.

(1a $S, 9$ a $S$ )-Dimethyl ester 1a,2,3,8,9,9a-hexahydro-4-(methoxymethoxy)-7-[(4methoxyphenyl)methyl]-9-oxo-1H-azirino[2,3-c][1]benzazocine-1,5-dicarboxylic acid.
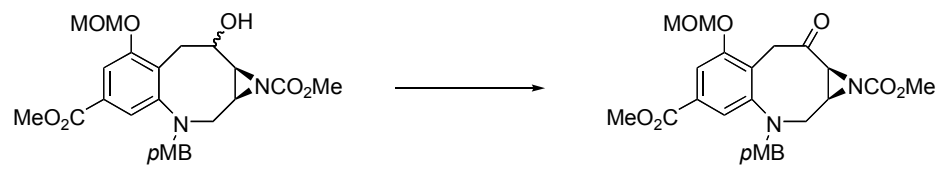

The alcohol from the previous reaction $(65 \mathrm{mg}, 0.134 \mathrm{mmol}, 1.0$ eq.) was dissolved in $4 \mathrm{~mL}$ of reagent grade $\mathrm{CH}_{2} \mathrm{Cl}_{2}$. Dess-Martin reagent $(85 \mathrm{mg}, 0.200 \mathrm{mmol}, 1.5$ eq.) was added in one portion and the reaction slowly turned a light purple color. The reaction was allowed to stir $1.5 \mathrm{hr}$. and was diluted with $\mathrm{Et}_{2} \mathrm{O}$ followed by a solution of $\mathrm{Na}_{2} \mathrm{~S}_{2} \mathrm{O}_{3}$ in sat. $\mathrm{NaHCO}_{3(\mathrm{aq})}$. The layers were vigorously stirred until clear. Following separation, the aqueous layer was extracted $4 \mathrm{X}$ EtOAC. The combined organic layers were washed $1 \mathrm{X} \mathrm{NaCl}_{(\mathrm{aq})}$, dried over $\mathrm{Na}_{2} \mathrm{SO}_{4}$, filtered, and concentrated. The crude product was purified by column chromatography $\left(2: 1 \mathrm{CH}_{2} \mathrm{Cl}_{2} / \mathrm{Et}_{2} \mathrm{O}\right)$ to give $48 \mathrm{mg}$ (75\% yield) of the ketone as a white crystalline solid following concentration with $\mathrm{Et}_{2} \mathrm{O}$.

$[\alpha]_{\mathrm{D}}^{25}=-45.6\left(\mathrm{c}=1.0, \mathrm{CHCl}_{3}\right)$ m.p. $58-62{ }^{\circ} \mathrm{C}{ }^{1} \mathrm{H} \mathrm{NMR}\left(300 \mathrm{MHz}, \mathrm{CDCl}_{3}\right) \delta \mathrm{CHCl}_{3}: 2.77(\mathrm{~m}, 1 \mathrm{H})$, $3.06(\mathrm{~d}, \mathrm{~J}=6.9 \mathrm{~Hz}, 1 \mathrm{H}), 3.35(\mathrm{~d}, \mathrm{~J}=3.0 \mathrm{~Hz}, 2 \mathrm{H}), 3.44(\mathrm{~s}, 3 \mathrm{H}), 3.46(\mathrm{~m}, 2 \mathrm{H}), 3.61(\mathrm{~s}, 3 \mathrm{H}), 3.76(\mathrm{~s}, 3 \mathrm{H})$, $3.91(\mathrm{~s}, 3 \mathrm{H}), 3.94(\mathrm{~m}, 2 \mathrm{H}), 5.20(1 / 2 \mathrm{ABq}, \mathrm{J}=6.6 \mathrm{~Hz}, 1 \mathrm{H}), 5.25(1 / 2 \mathrm{ABq}, \mathrm{J}=6.6 \mathrm{~Hz}, 1 \mathrm{H}), 6.78(\mathrm{~d}, \mathrm{~J}=$ $7.5 \mathrm{~Hz}, 2 \mathrm{H}), 7.08(\mathrm{~d}, \mathrm{~J}=2.5 \mathrm{~Hz}, 2 \mathrm{H}), 7.53(\mathrm{~d}, \mathrm{~J}=1.5 \mathrm{~Hz}, 1 \mathrm{H}), 7.56(\mathrm{~d}, \mathrm{~J}=1.5 \mathrm{~Hz}, 1 \mathrm{H}) ;{ }^{13} \mathrm{C}$ NMR $(75$ $\mathrm{MHz}_{2} \mathrm{CDCl}_{3}$ ) $\delta: ~ 39.1,45.5,46.3,52.3,52.4,53.7,55.1,56.2,56.3,60.6,94.2,112.0,113.5,118.4,128.2$, 129.7, 130.9, 131.1, 131.1,149.9, 153.8, 158.9, 161.7, 166.3, 200.0; IR (NaCl/neat) 2953, 1722, 1611, 1583 $\mathrm{cm}^{-1}$; HRMS (FAB+) calcd for $\mathrm{C}_{25} \mathrm{H}_{29} \mathrm{~N}_{2} \mathrm{O}_{8}(\mathrm{~m} / \mathrm{z})$ 485.1924, found $(\mathrm{m} / \mathrm{z})$ 485.1931.

(1aS,8R,9aS)-Dimethyl ester 1a,2,3,8,9,9a-hexahydro-8-(hydroxymethyl)-7-(methoxymethoxy)3-[(4-methoxyphenyl)methyl]-9-oxo-1H-azirino[2,3-c][1]benzazocine-1,5-dicarboxylic acid. (11)

(1aS,8S,9aS)-Dimethyl ester 1a,2,3,8,9,9a-hexahydro-8-(hydroxymethyl)-7-(methoxymethoxy)3-[(4-methoxyphenyl)methyl]-9-oxo-1H-azirino[2,3-c][1]benzazocine-1,5-dicarboxylic acid. (16)

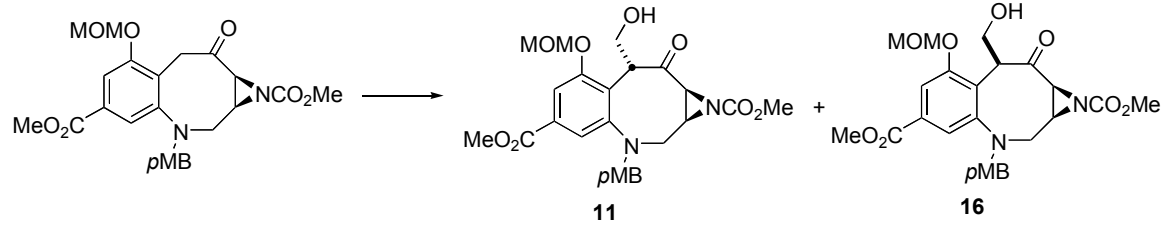

Ketone from the previous reaction ( $28 \mathrm{mg}, 57.8 \mu \mathrm{mol}, 1.0$ equiv.) was dissolved in $600 \mu \mathrm{L}$ of dry DMF and cooled to $-45^{\circ} \mathrm{C}$ over $20 \mathrm{~min}$. Next, a $0.86 \mathrm{M}$ solution of LDA (67.2 $\mu \mathrm{L}, 57.8 \mu \mathrm{mol}, 1.0$ equiv.) was added dropwise and the solution turned a deep orange. After stirring 15 min., a $0.2 \mathrm{M}$ solution of anhydrous formaldehyde in THF ( $491 \mu \mathrm{L}, 98.2 \mu \mathrm{mol}, 1.7$ equiv.) was added dropwise. The color of the solution faded to a light yellow over $15 \mathrm{~min}$. and the reaction was allowed to stir an additional $30 \mathrm{~min}$. at $45{ }^{\circ} \mathrm{C}$ before being quenched with a solution of sat. $\mathrm{NH}_{4} \mathrm{Cl}_{(\mathrm{aq})}$. The reaction was diluted with EtOAc and allowed to come to room temperature. The layers are separated and the aqueous layer was extracted $4 \mathrm{X}$ EtOAc. The combined organic layers are washed $1 \mathrm{X}$ brine, dried over $\mathrm{Na}_{2} \mathrm{SO}_{4}$, concentrated and dried overnight under vacuum. The product was purified by column chromatography $(3: 3: 1$ $\mathrm{CHCl}_{3}$ /benzene/acetone) to give $15 \mathrm{mg}$ (50\% yield) of alcohols $\mathbf{1 1}$ and $\mathbf{1 6}$ as a $1: 1$ mixture of 
diastereomers along with $10 \mathrm{mg}$ of recovered 16. The diastereomers were further separated by PTLC (2:2:1 $\mathrm{CHCl}_{3}$ /benzene/acetone) to furnish both $\mathbf{1 1}$ and $\mathbf{1 6}$ as clear colorless oils.

Compound 11: $[\alpha]_{\mathrm{D}}^{25}=-19.3\left(\mathrm{c}=1.8, \mathrm{CHCl}_{3}\right){ }^{1} \mathrm{H} \mathrm{NMR}\left(300 \mathrm{MHz}, \mathrm{CDCl}_{3}\right) \delta \mathrm{CHCl}_{3}: 3.02(\mathrm{dd}, \mathrm{J}=3.0 \mathrm{~Hz}$, $6.6 \mathrm{~Hz}, 1 \mathrm{H}), 3.17(\mathrm{~d}, \mathrm{~J}=6.6 \mathrm{~Hz}, 1 \mathrm{H}), 3.21(\mathrm{~m}, 2 \mathrm{H}), 3.48(\mathrm{~s}, 3 \mathrm{H}), 3.56(\mathrm{~s}, 3 \mathrm{H}), 3.80(\mathrm{~m}, 2 \mathrm{H}) 3.81(\mathrm{~s}, 3$ H), $3.86($ br d, J = 4.5 Hz, $2 \mathrm{H}), 3.92(\mathrm{~s}, 3 \mathrm{H}), 4.14(\mathrm{dd}, \mathrm{J}=4.5 \mathrm{~Hz}, 7.5 \mathrm{~Hz} 1 \mathrm{H}), 5.27(1 / 2 \mathrm{ABq}, \mathrm{J}=7.5,1$ $\mathrm{H}), 5.37(1 / 2 \mathrm{ABq}, \mathrm{J}=7.5,1 \mathrm{H}), 6.86(\mathrm{~d}, \mathrm{~J}=9.0,2 \mathrm{H}), 7.09(\mathrm{~d}, \mathrm{~J}=9.0,2 \mathrm{H}), 7.56(\mathrm{~d}, \mathrm{~J}=1.5 \mathrm{~Hz}, 1 \mathrm{H})$,

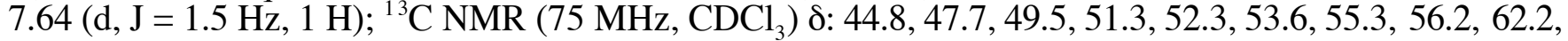
62.8, 93.8, 112.9, 113.8, 113.8, 119.2, 127.7, 130.3, 131.3, 131.3, 133.2, 150.4, 153.3, 159.1, 161.4, 166.1, 201.1; IR (NaCl/neat) 3530, 2954, 1724, 1678, $1583 \mathrm{~cm}^{-1}$; HRMS (FAB+) calcd for $\mathrm{C}_{26} \mathrm{H}_{31} \mathrm{~N}_{2} \mathrm{O}_{9}(\mathrm{~m} / z)$ 515.2030 found $(\mathrm{m} / \mathrm{z}) 515.2026$.

Compound: $16[\alpha]_{\mathrm{D}}^{25}=-41.3\left(\mathrm{c}=1.4, \mathrm{CHCl}_{3}\right){ }^{1} \mathrm{H} \mathrm{NMR}\left(300 \mathrm{MHz}, \mathrm{CDCl}_{3}\right) \delta \mathrm{CHCl}_{3}: 2.66(\mathrm{~m}, 1 \mathrm{H}), 3.08$ $(\mathrm{dd}, \mathrm{J}=3.0 \mathrm{~Hz}, 12.0 \mathrm{~Hz}, 1 \mathrm{H}), 3.11(\mathrm{~d}, \mathrm{~J}=6.0 \mathrm{~Hz}, 1 \mathrm{H}), 3.16(\mathrm{dd}, \mathrm{J}=6.0 \mathrm{~Hz}, 12.0 \mathrm{~Hz}, 1 \mathrm{H}), 3.52(\mathrm{~s}, 3 \mathrm{H})$, $3.66(\mathrm{~s}, 3 \mathrm{H}), 3.79(\mathrm{~s}, 3 \mathrm{H}), 3.56-3.83(\mathrm{~m}, 2 \mathrm{H}), 3.87(\mathrm{~m}, 2 \mathrm{H}), 3.95(\mathrm{~s}, 3 \mathrm{H}), 4.21(\mathrm{dd}, \mathrm{J}=3.0 \mathrm{~Hz}, 7.5 \mathrm{~Hz}$, $1 \mathrm{H}), 5.30(1 / 2 \mathrm{ABq}, \mathrm{J}=7.5,1 \mathrm{H}), 5.35(1 / 2 \mathrm{ABq}, \mathrm{J}=7.5,1 \mathrm{H}), 6.84(\mathrm{~d}, \mathrm{~J}=9.0 \mathrm{~Hz}, 2 \mathrm{H}), 7.07(\mathrm{~d}, \mathrm{~J}=9.0$ $\mathrm{Hz}, 2 \mathrm{H}), 7.73(\mathrm{~d}, \mathrm{~J}=1.5 \mathrm{~Hz}, 1 \mathrm{~Hz}), 7.77(\mathrm{~d}, \mathrm{~J}=1.5 \mathrm{~Hz}, 1 \mathrm{~Hz}) ;{ }^{13} \mathrm{C} \mathrm{NMR}\left(75 \mathrm{MHz}, \mathrm{CDCl}_{3}\right)$ 8: 43.2, 46.0, 50.0, 52.5, 53.4, 53.9, 55.2, 56.7, 61.3, 62.9, 94.4, 113.0, 113.9, 113.9, 119.9, 127.8, 130.3, 130.3, 130.4, 131.7, 150.3, 155.1, 159.0, 162.4, 165.8, 199.8; IR (NaCl/neat) 3525, 2954, 1724, $1514 \mathrm{~cm}^{-1}$; HRMS $(\mathrm{FAB}+)$ calcd for $\mathrm{C}_{26} \mathrm{H}_{31} \mathrm{~N}_{2} \mathrm{O}_{9}(\mathrm{~m} / z) 515.2030$ found $(\mathrm{m} / \mathrm{z}) 515.2034$.

(1aS,8R,9aS)-Dimethyl ester 1a,2,3,8,9,9a-hexahydro-8-(hydroxymethyl)-7-(methoxymethoxy)3-[(4-methoxyphenyl)methyl]-9-oxo-1H-azirino[2,3-c][1]benzazocine-1,5-dicarboxylic acid.
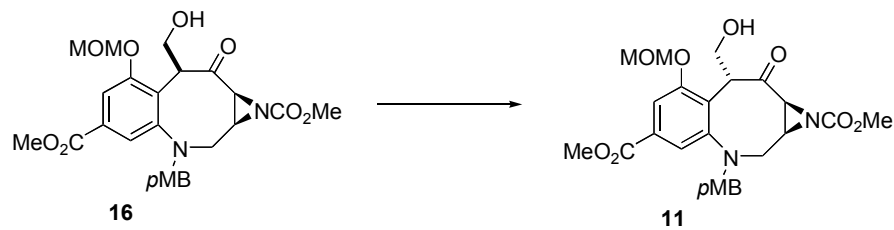

To a solution of alcohol $16(9.0 \mathrm{mg}, 17.5 \mu \mathrm{mol}, 1.0$ equiv.) in $4 \mathrm{~mL}$ of dry toluene was added 1,8diazabicyclo[5.4.0]undec-7-ene (DBU) $(16 \mu \mathrm{L}, 107.0 \mu \mathrm{mol}, 6.0$ equiv) and the reaction was stirred at ambient temperature. After $48 \mathrm{~h}$, no further progress of the reaction was observed by TLC analysis $(2: 2: 1$ $\mathrm{CH}_{3} \mathrm{Cl}$ /benzene/acetone) and sat. $\mathrm{NH}_{4} \mathrm{Cl}_{\text {(aq) }}$ was added to the mixture followed by dilution with $\mathrm{CH}_{2} \mathrm{Cl}_{2}$. The layers were separated and following dilution with $\mathrm{H}_{2} \mathrm{O}$ the aqueous layer was extracted $3 \mathrm{X} \mathrm{CH}_{2} \mathrm{Cl}_{2}$. The combined organic layers were washed $1 \mathrm{X}$ brine, dried over $\mathrm{Na}_{2} \mathrm{SO}_{4}$, filtered, and concentrated. Purification by PTLC (2:2:1 $\mathrm{CH}_{3} \mathrm{Cl} /$ benzene/acetone) afforded $6.3 \mathrm{mg}$ of alcohol 11 (70\% yield) along with $2.3 \mathrm{mg}$ of recovered starting material 16.

(1aS,8R,9aS)-Dimethyl ester 8-[[[(1,1-dimethylethyl)dimethylsilyl]oxy]methyl]-1a,2,3,8,9,9ahexahydro-7-(methoxymethoxy)-3-[(4-methoxyphenyl)methyl]-8-oxo-1H-azirino[2,3c][1]benzazocine-1,5-dicarboxylic acid.
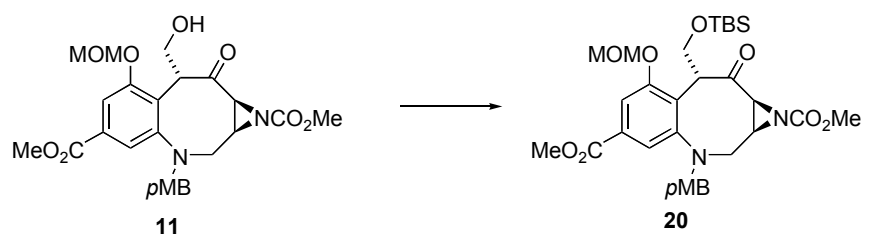
Alcohol 11 (34 mg, $66.1 \mu \mathrm{mol}, 1.0$ equiv.) was dissolved in $1 \mathrm{~mL}$ of $\mathrm{CH}_{2} \mathrm{Cl}_{2}$ and cooled to $-78{ }^{\circ} \mathrm{C}$ over $20 \mathrm{~min}$. Next, $213 \mu \mathrm{L}$ of a premixed solution of 1:1 TBSOTf ( $43 \mu \mathrm{L}, 187 \mu \mathrm{mol}, 2.8$ equiv. $)$ and 2,6 lutidine (24 $\mu \mathrm{L}, 206 \mu \mathrm{mol}, 3.1$ equiv.) in $\mathrm{CH}_{2} \mathrm{Cl}_{2}$ was added dropwise. The solution was allowed to warm to $0{ }^{\circ} \mathrm{C}$ and stirred at this temperature for $1.5 \mathrm{~h}$ after which TLC analysis $\left(2: 1 \mathrm{CH}_{2} \mathrm{Cl}_{2} / \mathrm{Et}_{2} \mathrm{O}\right)$ showed complete consumption of the starting material. The reaction was quenched with $\mathrm{NaHCO}_{3(\mathrm{aq})}$ followed by dilution with $\mathrm{CH}_{2} \mathrm{Cl}_{2}$ and $\mathrm{H}_{2} \mathrm{O}$ and the layers were separated. The aqueous phase was extracted $3 \mathrm{X} \mathrm{CH}_{2} \mathrm{Cl}_{2}$ and the combined organic layers washed $1 \mathrm{X} \mathrm{H}_{2} \mathrm{O}, 1 \mathrm{X}$ brine, and dried over $\mathrm{Na}_{2} \mathrm{SO}_{4}$. Concentration and purification by PTLC $\left(2: 1 \mathrm{CH}_{2} \mathrm{Cl}_{2} / \mathrm{Et}_{2} \mathrm{O}\right)$ afforded $40 \mathrm{mg}(96 \%$ yield) of product 20 as a clear colorless oil.

$[\alpha]_{\mathrm{D}}^{25}=-18.8\left(\mathrm{c}=1.7, \mathrm{CHCl}_{3}\right){ }^{1} \mathrm{H}$ NMR $(300 \mathrm{MHz}$, toluene-d6, $323 \mathrm{~K}) \delta: 0.04(\mathrm{~s}, 3 \mathrm{H}), 0.09(\mathrm{~s}, 3 \mathrm{H}), 0.89$ $(\mathrm{s}, 9 \mathrm{H}), 2.38(\mathrm{dd}, \mathrm{J}=2.5 \mathrm{~Hz}, 6.5 \mathrm{~Hz}, 1 \mathrm{H}), 2.77(\mathrm{dd}, \mathrm{J}=3.0 \mathrm{~Hz}, 14.0 \mathrm{~Hz}, 1 \mathrm{H}), 2.85(\mathrm{~d}, \mathrm{~J}=14.0 \mathrm{~Hz}, 1 \mathrm{H})$, $2.95(\mathrm{~d}, \mathrm{~J}=6.5 \mathrm{~Hz}, 1 \mathrm{H}), 3.07(\mathrm{~s}, 3 \mathrm{H}), 3.23(\mathrm{~s}, 3 \mathrm{H}), 3.34(\mathrm{~s}, 3 \mathrm{H}), 3.51(\mathrm{~s}, 3 \mathrm{H}), 3.68(\mathrm{~d}, \mathrm{~J}=13.0 \mathrm{~Hz}, 1$ H), $3.87(\mathrm{~d}, \mathrm{~J}=13.0 \mathrm{~Hz}, 1 \mathrm{H}), 4.11(\mathrm{dd}, \mathrm{J}=2.5 \mathrm{~Hz}, 8.5 \mathrm{~Hz}, 1 \mathrm{H}), 4.15(\mathrm{dd}, \mathrm{J}=2.5 \mathrm{~Hz}, 7.5 \mathrm{~Hz}, 1 \mathrm{H}), 4.22$, $(\mathrm{dd}, \mathrm{J}=5.0 \mathrm{~Hz}, 8.0 \mathrm{~Hz}, 1 \mathrm{H}), 4.86(\mathrm{~d}, \mathrm{~J}=6.5 \mathrm{~Hz}, 1 \mathrm{H}), 4.96(\mathrm{~d}, \mathrm{~J}=6.5 \mathrm{~Hz}, 1 \mathrm{H}), 6.79(\mathrm{~d}, \mathrm{~J}=8.5 \mathrm{~Hz}, 2 \mathrm{H})$,

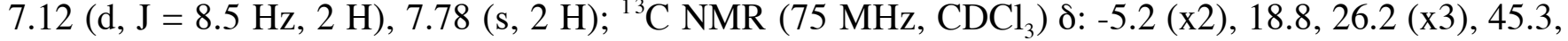
47.2, 49.4, 51.5, 52.4, 53.7, 55.4, 56.3, 61.6, 61.8, 94.3, 113.0, 113.8, 113.8, 119.4, 128.7, 130.1, 131.9, 131.9, 134.7, 151.7, 153.9, 159.3, 162.0, 166.7, 197.8; IR (NaCl/neat) 2953, 1726, 1692, $1514 \mathrm{~cm}^{-1}$; HRMS (FAB+) calcd for $\mathrm{C}_{32} \mathrm{H}_{45} \mathrm{~N}_{2} \mathrm{O}_{9} \mathrm{Si}_{1}(\mathrm{~m} / z) 629.2894$ found $(\mathrm{m} / \mathrm{z}) 629.2896$.

$(1 \mathrm{a} S, 3 R, 8 R, 9 R, 9 \mathrm{a} S)$-Dimethyl ester 8-[[[(1,1 dimethylethyl)dimethylsilyl]oxy]methyl]1,1a,2,8,9,9a-hexahydro-9-hydroxy-7-(methoxymethoxy)-3,9-epoxy-3H-azirino[2,3c][1]benzazocine-1,5-dicarboxylic acid.

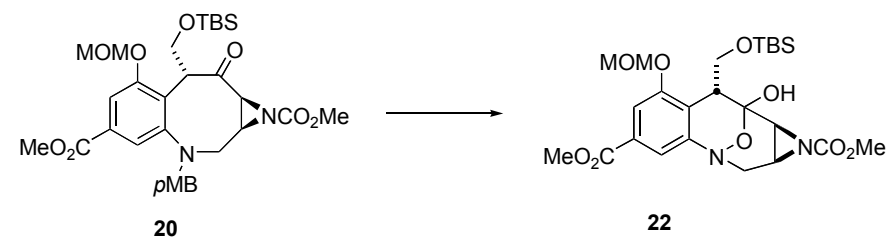

Compound 20 ( $25 \mathrm{mg}, 39.7 \mu \mathrm{mol}, 1.0$ equiv.) was dissolved in $2 \mathrm{~mL}$ of $\mathrm{CH}_{2} \mathrm{Cl}_{2}$ and cooled to $0{ }^{\circ} \mathrm{C}$ over 20 min. Next, $2 \mathrm{~mL}$ of sat. $\mathrm{K}_{2} \mathrm{CO}_{3(\mathrm{ag})}$ was added and the mixture was stirred for 5 min after which 3.5 $\mathrm{mL}$ of a freshly prepared solution of DMDO at $-20{ }^{\circ} \mathrm{C}$ (0.8-0.9 $\mathrm{M}$, determined by titration with $\mathrm{PPh}_{3}$ according to Murray et $a .^{23 \mathrm{~b}}$ ) was added via a cold syringe. The resulting biphasic mixture was protected from light and allowed to slowly come to room temperature over $6.5 \mathrm{~h}$ with vigorous stirring. The reaction was poured into a separatory funnel and the layers were separated. Dilution of the aqueous layer with $\mathrm{H}_{2} \mathrm{O}$ was followed by extraction with $3 \mathrm{X} \mathrm{CH}_{2} \mathrm{Cl}_{2}$. The combined organic extracts were washed $3 \mathrm{X}$ sodium phosphate buffer $(\mathrm{pH}=6), 1 \mathrm{X}$ brine and dried over $\mathrm{Na}_{2} \mathrm{SO}_{4}$. Concentration afforded a crude yellow oil that was purified by PTLC $\left(2: 1 \mathrm{CH}_{2} \mathrm{Cl}_{2} / \mathrm{Et}_{2} \mathrm{O}\right)$ to give $9.0 \mathrm{mg}$ of hydroxylamine hemi-ketal 22 (43\% yield) as a light yellow oil along with recovery of $12 \mathrm{mg}$ of unreacted ketone $\mathbf{2 0}$.

$[\alpha]_{\mathrm{D}}^{25}=+8.0\left(\mathrm{c}=0.48, \mathrm{CHCl}_{3}\right)^{1} \mathrm{H} \mathrm{NMR}\left(400 \mathrm{MHz} \mathrm{CDCl}_{3}\right) \delta \mathrm{CHCl}_{3}: \quad 0.16(\mathrm{~s}, 3 \mathrm{H}), 0.21(\mathrm{~s}, 3 \mathrm{H}), 0.97$ $(\mathrm{s}, 9 \mathrm{H}), 2.76(\mathrm{dd}, \mathrm{J}=2.0 \mathrm{~Hz}, 6.0 \mathrm{~Hz}, 1 \mathrm{H}), 3.21(\mathrm{~d}, \mathrm{~J}=6.0 \mathrm{~Hz}, 1 \mathrm{H}), 3.48(\mathrm{~s}, 3 \mathrm{H}), 3.47-3.51(\mathrm{~m}, 1 \mathrm{H})$, $3.58(\mathrm{~s}, 3 \mathrm{H}), 3.68(\mathrm{~d}, \mathrm{~J}=14.0 \mathrm{~Hz}, 1 \mathrm{H}), 3.89(\mathrm{~s}, 3 \mathrm{H}), 3.93(\mathrm{dd}, \mathrm{J}=2.0 \mathrm{~Hz}, 14.0 \mathrm{~Hz}, 1 \mathrm{H}), 4.00(\mathrm{dd}, \mathrm{J}=$ $10.4, \mathrm{~J}=10.4,1 \mathrm{H}), 4.11(\mathrm{dd}, \mathrm{J}=4.0 \mathrm{~Hz}, 10.4 \mathrm{~Hz}, 1 \mathrm{H}), 5.24(1 / 2 \mathrm{ABq}, \mathrm{J}=6.0 \mathrm{~Hz}, 1 \mathrm{H}), 5.34(1 / 2 \mathrm{ABq}, \mathrm{J}$ $=6.0 \mathrm{~Hz}, 1 \mathrm{H}), 7.12(\mathrm{~d}, \mathrm{~J}=1.2 \mathrm{~Hz}, 1 \mathrm{H}), 7.36(\mathrm{~d}, \mathrm{~J}=1.6 \mathrm{~Hz}, 1 \mathrm{H}), 7.84(\mathrm{~s}, 1 \mathrm{H}) ;{ }^{13} \mathrm{C} \mathrm{NMR}(100 \mathrm{MHz}$,

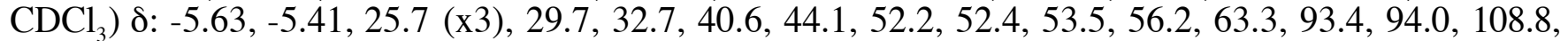


113.3, 119.3, 129.8, 149.3, 153.9, 161.8, 166.5; IR (NaCl/neat) 3339, 2926, 1726, $1584 \mathrm{~cm}^{-1}$; HRMS (FAB+) calcd for $\mathrm{C}_{24} \mathrm{H}_{37} \mathrm{~N}_{2} \mathrm{O}_{9} \mathrm{Si}_{1}(\mathrm{~m} / \mathrm{z}) 525.2268$ found $(\mathrm{m} / \mathrm{z}) 525.2261$.

$(1 \mathrm{a} S, 3 R, 8 R, 9 R, 9 \mathrm{a} S)$-Dimethyl ester 1,1a,2,8,9,9a-hexahydro-9-hydroxy-8-(hydroxymethyl)-7(methoxymethoxy)-3,9-epoxy-3H-azirino[2,3-c][1]benzazocine-1,5-dicarboxylic acid.

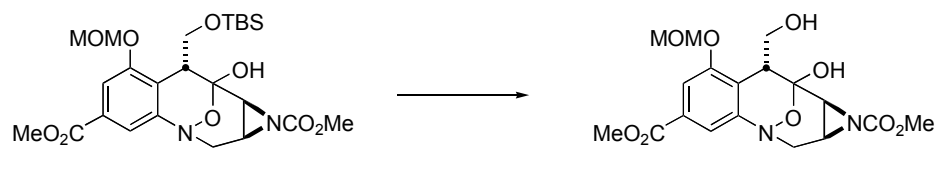

Compound 22 ( $7 \mathrm{mg}, 13.3 \mu \mathrm{mol}, 1.0$ equiv.) was dissolved in $500 \mu \mathrm{L}$ of THF and cooled to $0{ }^{\circ} \mathrm{C}$ over 20 min. To the resulting solution was added $1 \mathrm{M}$ TBAF in THF (23 $\mu \mathrm{L}, 23.0 \mu \mathrm{mol} .1 .7$ equiv.) and the solution was stirred for $1 \mathrm{~h}$ after which TLC analysis $\left(20: 1 \mathrm{CH}_{2} \mathrm{Cl}_{2} / \mathrm{MeOH}\right)$ revealed complete consumption of the starting material. The reaction was quenched with sat. $\mathrm{NH}_{4} \mathrm{Cl}_{\text {(aq) }}$, stirred vigorously for 10 min., and diluted with EtOAc and $\mathrm{H}_{2} \mathrm{O}$. The layers were partitioned and the aqueous layer was extracted with $3 \mathrm{X} \mathrm{CH}_{2} \mathrm{Cl}_{2}$. The combined organic layers were washed $1 \mathrm{X}$ brine and dried over $\mathrm{Na}_{2} \mathrm{SO}_{4}$. Purification by PTLC (20:1 $\left.\mathrm{CH}_{2} \mathrm{Cl}_{2} / \mathrm{MeOH}\right)$ afforded $5 \mathrm{mg}$ (92\% yield) of the alcohol as a colorless oil.

$[\alpha]_{\mathrm{D}}^{25}=+10.5\left(\mathrm{c}=0.40, \mathrm{CHCl}_{3}\right){ }^{1} \mathrm{H} \mathrm{NMR}\left(300 \mathrm{MHz}, \mathrm{CDCl}_{3}\right) \delta \mathrm{CHCl}_{3}: 2.55$ (s, br, $\left.1 \mathrm{H}\right), 2.78(\mathrm{dd}, \mathrm{J}=$ $2.1 \mathrm{~Hz}, 6.9 \mathrm{~Hz}, 1 \mathrm{H}), 3.22(\mathrm{~d}, \mathrm{~J}=6.9, \mathrm{~Hz}, 1 \mathrm{H}), 3.48(\mathrm{~s}, 3 \mathrm{H}), 3.55(\mathrm{~m}, 1 \mathrm{H}), 3.58(\mathrm{~s}, 3 \mathrm{H}), 3.68(\mathrm{~d}, \mathrm{~J}=15.0$ $\mathrm{Hz}, 1 \mathrm{H}), 3.89(\mathrm{~s}, 3 \mathrm{H}), 3.94(\mathrm{~d}, \mathrm{~J}=2.1 \mathrm{~Hz}, 15.0 \mathrm{~Hz}), 4.03(\mathrm{dd}, \mathrm{J}=12.0 \mathrm{~Hz}, 12.0 \mathrm{~Hz}, 1 \mathrm{H}), 4.17$, (dd, J = 3.0 Hz, 12.0 Hz, 1 H), $5.25(1 / 2 \mathrm{ABq}, \mathrm{J}=6.0,1 \mathrm{H}), 5.35(1 / 2 \mathrm{ABq}, \mathrm{J}=6.0,1 \mathrm{H}), 6.95(\mathrm{~s}, \mathrm{br}, 1 \mathrm{H}), 7.13(\mathrm{~d}$,

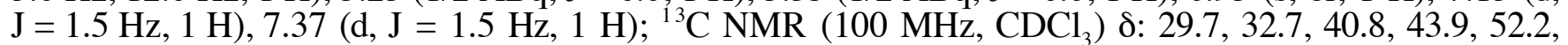
53.6, 56.2, 62.8, 93.4, 94.0, 109.0, 113.2, 119.4, 129.9, 149.1, 153.9, 161.7, 166.4; IR (NaCl/neat) 3331, 2924, 1717, $1684 \mathrm{~cm}^{-1}$; HRMS (FAB+) calcd for $\mathrm{C}_{18} \mathrm{H}_{22} \mathrm{~N}_{2} \mathrm{O}_{9}(\mathrm{~m} / \mathrm{z}) 410.1325$ found $(\mathrm{m} / \mathrm{z}) 410.1314$.

$(1 \mathrm{a} S, 3 R, 8 R, 9 R, 9 \mathrm{a} S)$-Dimethyl ester 8-[[(aminocarbonyl)oxy]methyl]-1,1a,2,8,9,9a-hexahydro9-hydroxy-7-(methoxymethoxy)-3,9-epoxy-3H-azirino[2,3-c][1]benzazocine-1,5-dicarboxylic acid.
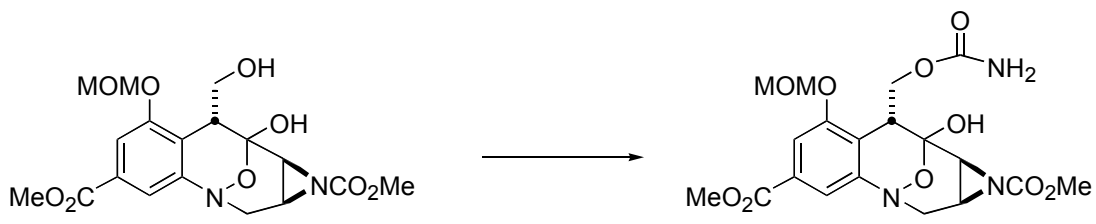

25

To a solution of the alcohol $\left(6.5 \mathrm{mg}, 15.8 \mu \mathrm{mol}, 1.0\right.$ equiv.) in $600 \mu \mathrm{L} \mathrm{CH}_{2} \mathrm{Cl}_{2}$ at $0{ }^{\circ} \mathrm{C}$ was added trichloroacetyl isocyanate $(5 \mu \mathrm{L}, 42.0 \mu \mathrm{mol}, 2.7$ equiv.). The reaction was stirred $5 \mathrm{~min}$ and quenched with the addition of 5 drops of $\mathrm{MeOH}$. The reaction was concentrated in vacou and the crude intermediate was dissolved in $3 \mathrm{~mL}$ of $\mathrm{MeOH}$. Silica gel was added to give a slurry and the mixture was stirred for $8 \mathrm{~h}$ at ambient temperature. The reaction was filtered with $10: 1 \mathrm{CH}_{2} \mathrm{Cl}_{2} / \mathrm{MeOH}$ and concentrated. Purification of the crude mixture by PTLC $\left(20: 1 \mathrm{CH}_{2} \mathrm{Cl}_{2} / \mathrm{MeOH}\right)$ furnished $6.2 \mathrm{mg}$ (86\% yield) of carbamate 25 as a white amorphous solid.

$[\alpha]_{\mathrm{D}}^{25}=-29.7\left(\mathrm{c}=0.70, \mathrm{CHCl}_{3}\right){ }^{1} \mathrm{H}$ NMR $\left(300 \mathrm{MHz}, \mathrm{CDCl}_{3}\right) \delta \mathrm{CHCl}_{3}: 2.77(\mathrm{dd}, \mathrm{J}=2.0 \mathrm{~Hz}, 6.5 \mathrm{~Hz}, 1 \mathrm{H})$, $3.19(\mathrm{~d}, \mathrm{~J}=6.5, \mathrm{~Hz}, 1 \mathrm{H}), 3.33(\mathrm{~d}, \mathrm{~J}=3.5 \mathrm{~Hz}, 1 \mathrm{H}), 3.48(\mathrm{~s}, 3 \mathrm{H}), 3.57(\mathrm{~s}, 3 \mathrm{H}), 3.71(\mathrm{~d}, \mathrm{~J}=6.5 \mathrm{~Hz}, 1 \mathrm{H})$, $3.86(\mathrm{~m}, 1 \mathrm{H}), 3.90(\mathrm{~s}, 3 \mathrm{H}), 4.59(\mathrm{dd}, \mathrm{J}=4.0 \mathrm{~Hz}, 12.5 \mathrm{~Hz}, 1 \mathrm{H}), 4.78(\mathrm{~s}, \mathrm{br}, 2 \mathrm{H}), 5.09$ (d, J = 12.5 Hz, 1 H), $5.29(\mathrm{~d}, \mathrm{~J}=6.5 \mathrm{~Hz}, 1 \mathrm{H}), 5.35(\mathrm{~d}, \mathrm{~J}=6.5 \mathrm{~Hz}, 1 \mathrm{H}), 6.92(\mathrm{~s}, \mathrm{br}, 1 \mathrm{H}), 7.15(\mathrm{~d}, \mathrm{~J}=1.5 \mathrm{~Hz}, 1 \mathrm{H}), 7.41(\mathrm{~d}$,

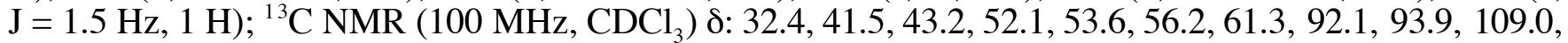


113.4, 120.3, 129.8, 149.7, 153.8, 158,6, 161.7, 166.5; IR (NaCl/neat) 3359, 2954, 1722, $1584 \mathrm{~cm}^{-1}$; HRMS (FAB+) calcd for $\mathrm{C}_{18} \mathrm{H}_{23} \mathrm{~N}_{3} \mathrm{O}_{9}(\mathrm{~m} / \mathrm{z}) 453.1383$ found $(\mathrm{m} / \mathrm{z}) 453.1314$.

$(1 \mathrm{a} S, 3 R, 8 R, 9 R, 9 \mathrm{a} S)$ - dimethyl ester 8-[[(aminocarbonyl)oxy]methyl]-1,1a,2,8,9,9a-hexahydro7,9-dihydroxy-3,9-epoxy-3H-azirino[2,3-c][1]benzazocine-1,5-dicarboxylic acid.
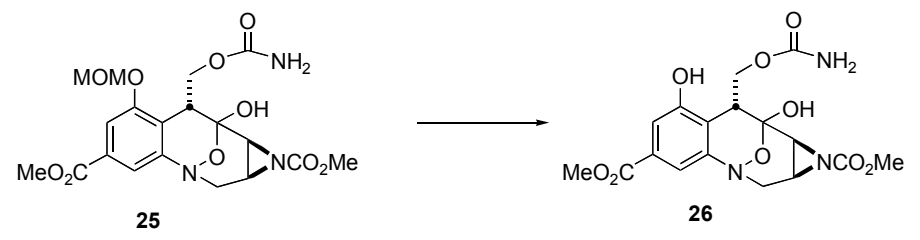

Compound 25 ( $8.5 \mathrm{mg}, 18.8 \mu \mathrm{mol}, 1.0$ equiv.) was dissolved in $600 \mu \mathrm{L}$ of $\mathrm{CH}_{2} \mathrm{Cl}_{2}$ and cooled to $-45{ }^{\circ} \mathrm{C}$ over 20 min. TMSBr (15 $\mu \mathrm{L}, 114 \mu \mathrm{mol}, 6.0$ equiv.) was added dropwise and the solution was stirred $1.0 \mathrm{~h}$ while maintaining a reaction temperature between -45 and $-40{ }^{\circ} \mathrm{C}$. An additional portion of $\mathrm{TMSBr}(15 \mu \mathrm{L}$, $114 \mu \mathrm{mol}, 6.0$ equiv.) was added and the reaction was continued for $2 \mathrm{~h}$. The reaction was quenched with $\mathrm{NaHCO}_{3(\mathrm{aq})}$ and allowed to come to ambient temperature. The mixture was diluted with $\mathrm{CH}_{2} \mathrm{Cl}_{2}$ and the layers were separated. Sat. $\mathrm{NH}_{4} \mathrm{Cl}_{\text {(aq) }}$ was added to the aqueous portion until a $\mathrm{pH}<9$ was obtained and the resulting solution was extracted $1 \mathrm{X} \mathrm{CH}_{2} \mathrm{Cl}_{2}$ and $3 \mathrm{X}$ THF. The combined organic layers were washed $1 \mathrm{X}$ brine, dried over $\mathrm{Na}_{2} \mathrm{SO}_{4}$, and concentrated. Purification by PTLC $\left(10: 1 \mathrm{CH}_{2} \mathrm{Cl}_{2} / \mathrm{MeOH}\right)$ afforded $4.6 \mathrm{mg}$ (60\% yield) of phenol $\mathbf{2 6}$ as a white solid.

$[\alpha]_{\mathrm{D}}^{25}=+10.5\left(\mathrm{c}=0.42, \mathrm{CHCl}_{3}\right){ }^{1} \mathrm{H} \mathrm{NMR}\left(300 \mathrm{MHz}, \mathrm{CDCl}_{3}\right) \delta \mathrm{CHCl}_{3}: 2.77(\mathrm{dd}, \mathrm{J}=2.0 \mathrm{~Hz}, 6.5 \mathrm{~Hz}, 1 \mathrm{H})$, $3.20(\mathrm{~d}, \mathrm{~J}=6.5 \mathrm{~Hz}, 1 \mathrm{H}), 3.39(\mathrm{~d}, \mathrm{~J}=5.5 \mathrm{~Hz}, 1 \mathrm{H}), 3.60(\mathrm{~s}, 3 \mathrm{H}), 3.69(\mathrm{~d}, \mathrm{~J}=14.5 \mathrm{~Hz}, 1 \mathrm{H}), 3.87(\mathrm{~m}, 1 \mathrm{H})$, $3.89(\mathrm{~s}, 3 \mathrm{H}), 4.50(\mathrm{dd}, \mathrm{J}=5.5 \mathrm{~Hz}, 13.0 \mathrm{~Hz}, 1 \mathrm{H}), 4.79$ (d, J = 13.0 Hz, $1 \mathrm{H}), 4.7-4.9$ (s, br, $2 \mathrm{H}), 5.79$ (s, br, $1 \mathrm{H}), 6.74(\mathrm{~s}, \mathrm{br}, 1 \mathrm{H}), 7.03(\mathrm{~d}, \mathrm{~J}=2.0 \mathrm{~Hz}, 1 \mathrm{H}), 7.19(\mathrm{~d}, \mathrm{~J}=2.0 \mathrm{~Hz}, 1 \mathrm{H}) ;{ }^{13} \mathrm{C} \mathrm{NMR}(100 \mathrm{MHz}$, $\left.\mathrm{CDCl}_{3}\right)$ \&: 32.6, 41.0, 43.2, 52.2, 53.9, 62.2, 92.4, 111.4, 111.6, 117.5, 129.6, 149.1, 154.1, 158.8, 162.0, 166.9; IR (NaCl/neat) 3363, 2917, 2849, 1701, $1539 \mathrm{~cm}^{-1}$; HRMS (FAB+) calcd for $\mathrm{C}_{16} \mathrm{H}_{23} \mathrm{~N}_{3} \mathrm{O}_{8}(\mathrm{~m} / \mathrm{z})$ 410.1199 found $(\mathrm{m} / \mathrm{z}) 410.1199$.

Synthesis of FR66979 (2).
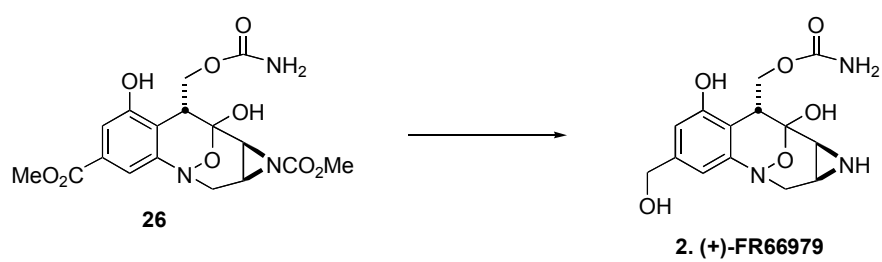

To a solution of compound 26 (4.0 mg, $9.8 \mu \mathrm{mol}, 1.0$ equiv.) in $400 \mu \mathrm{L}$ THF were added a $2 \mathrm{M} \mathrm{LiBH}_{4}$ solution in THF $(30 \mu \mathrm{L}, 60 \mu \mathrm{mol}, 6.1$ equiv.) and dry MeOH $(2.4 \mu \mathrm{L}, 59 \mu \mathrm{mol}, 6.0$ equiv.). The reaction was stirred at ambient temperature and following $1 \mathrm{~h}$ an identical aliquot of $\mathrm{LiBH}_{4}$ solution and $\mathrm{MeOH}$ were added. The additions were continued with two more aliquots every hour followed by two additional portions every three hours for a total of nine hours. Following the additions, the reaction was continued for nine more hours. The reaction was quenched with sat. $\mathrm{NH}_{4} \mathrm{Cl}_{(\mathrm{aq})}$ and stirred for $30 \mathrm{~min}$. The THF was concentrated off under reduced pressure and the remaining aqueous mixture was passed through a $\mathrm{C}_{8}$ Sep Pak (Waters) pre-washed with $1 \mathrm{X} \mathrm{MeOH}, 3 \mathrm{X} \mathrm{H}_{2} \mathrm{O}$. The Sep Pak was washed $3 \mathrm{X} \mathrm{H}_{2} \mathrm{O}$ and the product was eluded off with $\mathrm{MeOH}$. Next, $10 \% \mathrm{Pd} / \mathrm{C}(\sim 15 \mathrm{mg})$ was added to the $\mathrm{MeOH}$ filtrate and the mixture was stirred for $12 \mathrm{hrs}$. Filtration through a celite pad with hot $\mathrm{MeOH}$ and concentration gave crude FR66979 which was purified by PTLC $\left(9: 4 \mathrm{CH}_{2} \mathrm{Cl}_{2} / \mathrm{MeOH}\right.$, eluted off plate with 2.5:1 $\left.\mathrm{CH}_{2} \mathrm{Cl}_{2} / \mathrm{MeOH}\right)$ to 
furnish $2.7 \mathrm{mg}$ of (+)-FR66979 as a white solid. The synthetic and natural samples had identical nmr, IR, mass spec and mobility on TLC.

$[\alpha]_{\mathrm{D}}^{25}=+9.4\left(\mathrm{c}=0.17, \mathrm{H}_{2} \mathrm{O}\right) *{ }^{1} \mathrm{H}$ NMR $\left(400 \mathrm{MHz}, \mathrm{CD}_{3} \mathrm{OD}\right) \delta \mathrm{CD}_{3} \mathrm{OH}: 2.45-2.80(\mathrm{br}, 2 \mathrm{H}), 3.66$ (br, 2 H), $3.69(\mathrm{~m}, 1 \mathrm{H}), 4.47(\mathrm{~s}, 2 \mathrm{H}), 4.68(\mathrm{~d}, \mathrm{~J}=11.2 \mathrm{~Hz}, 1 \mathrm{H}), 5.06(\mathrm{dd}, \mathrm{J}=6.4 \mathrm{~Hz}, 11.2 \mathrm{~Hz}, 1 \mathrm{H}), 6.34(\mathrm{~s}, 1$ $\mathrm{H}), 6.51(\mathrm{~s}, 1 \mathrm{H}), 8.57(\mathrm{br}, 1 \mathrm{H})$; IR (NaCl/neat) 3302, 2924, 1709, $1587 \mathrm{~cm}^{-1}$; MS (ES) (\% Total ion count) $346(\mathrm{M}+\mathrm{Na}, 38), 324(\mathrm{M}+\mathrm{H}, 40)$.

*Note: for synthetic FR66979, $[\alpha]_{\mathrm{D}}^{25}=+9.4\left(\mathrm{c} 0.17, \mathrm{H}_{2} \mathrm{O}\right)$, lit. $=+12.5\left(\mathrm{c} 0.87, \mathrm{H}_{2} \mathrm{O}\right)$. On the polarimeter used in our laboratory, natural FR66979 exhibited an $[\alpha]_{\mathrm{D}}^{25}=+11.5\left(\mathrm{c} 0.22, \mathrm{H}_{2} \mathrm{O}\right)$. The optical activity of aziridine 11 was determined to be 93.5 : 6.5 er reflecting the Sharpless asymmetric epoxidation step.

\section{Synthesis of (+)-FR900482 (1).}
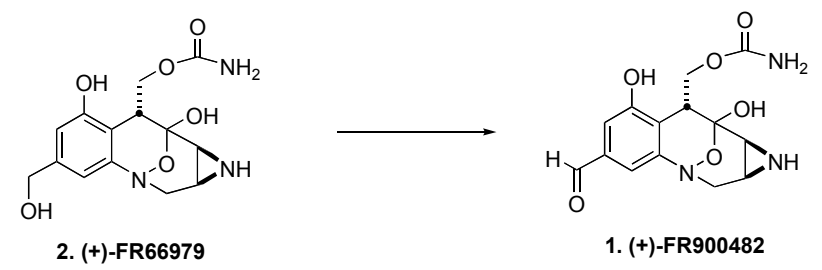

Oxalyl chloride $(10.4 \mu \mathrm{L}, 119.2 \mu \mathrm{mol}, 4.5$ eq.) was added to $417 \mu \mathrm{L}$ of THF in a $5 \mathrm{~mL}$ flask cooled to $78{ }^{\circ} \mathrm{C}$. Following the addition, $122 \mu \mathrm{L}$ of a stock solution consisting of $190 \mu \mathrm{L}$ of DMSO in $1.2 \mathrm{~mL}$ of THF was added dropwise and the resulting solution was warmed to $-35^{\circ} \mathrm{C}$ for approximately 3 min. and then cooled back to $-78^{\circ} \mathrm{C}$. A solution of FR66979 (2) $(8.5 \mathrm{mg}, 26.3 \mu \mathrm{mol}, 1.0$ eq.) in $418 \mu \mathrm{L}$ of a $4: 1$ solution of DMSO/THF was added dropwise and the solution was allowed to warm to $-35{ }^{\circ} \mathrm{C}$ for $15 \mathrm{~min}$. Next, $\mathrm{Et}_{3} \mathrm{~N}\left(48 \mu \mathrm{L}, 344.4 \mu \mathrm{mol}, 13.1\right.$ eq.) was added and the reaction was stirred at $0{ }^{\circ} \mathrm{C}$ for $20 \mathrm{~min}$. before being quenched by the addition of brine. Following dilution with THF, the reaction was stirred vigorously and separated. The aqueous layer was extracted $3 \mathrm{x}$ THF and the combined organic layers were washed 1X brine, dried over $\mathrm{Na}_{2} \mathrm{SO}_{4}$, and concentrated. After drying under vacuum $24 \mathrm{hrs}$., the crude material was purified by PTLC using 9:4 $\mathrm{CH}_{2} \mathrm{Cl}_{2} / \mathrm{MeOH}$ to give $2.6 \mathrm{mg}$ of FR900482 (1) in $33 \%$ yield along with $50 \%$ recovered FR66979 (2). The spectroscopic and analytical data matched that of the natural material in all respects.

1. (a) DNA Cross-link Formation by a Phototriggered Mitosene Progenitor. Judd, T.; Williams, R.M., Org. Lett. 2002, 4, 3711 3714; (b) Synthesis of the First Photo-triggered Pro-Mitosene Based on FR-900482. Williams, R.M.; Rollins, S.B.; Judd, T.C., Tetrahedron, 2000, 56, 521 532.

2. The purity of all new compounds was determined to be $>99 \%$ by ${ }^{1} \mathrm{H}$ nmr and HPLC analysis. 